\title{
Expression of Secreted Protein Acidic and Rich in Cysteine in the Stroma of a Colorectal Carcinoma is Associated With Patient Prognosis
}

\author{
Jeong Yeon Kim, Dongjun Jeong, Tae Sung Ahn', Hyung Ju Kim, Doo San Park, So Yong Park, \\ Sang Byung Bae ${ }^{2}$, Sookyoung Lee, Sung Soo Lee ${ }^{3}$, Moon Soo Lee ${ }^{1}$, Hyun Deuk Cho, Moo Jun Baek ${ }^{1}$ \\ Departments of Pathology, ${ }^{1}$ Surgery, ${ }^{2}$ Internal Medicine, and ${ }^{3}$ Preventive Medicine, Soonchunhyang University College of Medicine, Cheonan, \\ Korea
}

Purpose: Secreted protein acidic and rich in cysteine (SPARC), also known as osteonectin or basement-membrane-40 (BM40 ), is a member of a family of matricellular proteins, whose functions are to modulate cell-matrix interactions, growth and angiogenesis in colorectal cancer. In this study, the expression of SPARC was evaluated and its correlations with clinicopathological parameters were investigated.

Methods: The researchers analyzed the expression patterns of SPARC by using immunohistochemistry in 332 cases of colorectal cancer of tissue microarray. The clinicopathological characteristics were defined by using the TNM criteria of the Union for International Cancer Control. Clinicopathological factors such as age, sex, histologic type of the tumor, pathologic tumor stage, TNM stage, and lymphovascular invasion were evaluated according to the SPARC expression.

Results: The hazard ratios expressing SPARC in tumor cells, in the stroma, and in both tumor cells and the stroma were $2.10(\mathrm{P}=0.036), 3.27(\mathrm{P}=0.003)$ and $2.12(\mathrm{P}=0.038)$, respectively. Patient survival was decreased in patient expressing SPARC in the stroma, and this result showed statistical significance $(\mathrm{P}=0.016)$.

Conclusion: These findings suggest that SPARC expression in a tumor and in the stroma correlates with disease progression and may be used as a prognostic marker for colorectal cancer.

Keywords: SPARC; Osteonectin; Colorectal neoplasms; Prognosis

\section{INTRODUCTION}

Colorectal cancer, a kind of malignant tumor, has been increasing in Korea due to westernized eating habits, so more diversified research on tumor generation and advancement is needed. The growth and development of a cancer is influenced a lot by its surrounding environment. Secreted protein acidic and rich in cyste-

Received: March 20, 2013 - Accepted: April 17, 2013

Correspondence to: Moo Jun Baek, M.D.

Department of Surgery, Soonchunhyang University Cheonan Hospital,

Soonchunhyang University College of Medicine, 31 Suncheonhyang 6-gil, Dongnam-gu, Cheonan 330-930, Korea

Tel: +82-41-570-3633, Fax: +82-41-571-0129, E-mail: ssurge@sch.ac.kr

(C) 2013 The Korean Society of Coloproctology

This is an open-access article distributed under the terms of the Creative Commons Attribution NonCommercial License (http://creativecommons.org/licenses/by-nc/3.0) which permits unrestricted non-

commercial use, distribution, and reproduction in any medium, provided the original work is properly cited. ine (SPARC, also called osteonectin or basement-membrane-40 [BM-40]) is one of the matricellular protein groups that play the role of inducing interaction between and cell function in cells and substrates [1]. This protein gene is located at chromosome $5 \mathrm{q} 31$ 33 , and the human SPARC is composed of 10 exons. The structures of this gene in organisms from drosophila to higher animals are very similar. The cancer-related action mechanism of SPARC varies depending on the tumor type, and SPARC is known to be more severely expressed in the stroma around the tumor than in the tumor cell. SPARC has been reported to appear in breast cancer, melanoma, glioblastoma, ovarian cancer, pancreatic cancer, acute myelogenous leukemia, and colorectal cancer [2-10].

Over the past few years, most of the cancer research has been focused on the genetic change in the process of conversion into malignant cells. However, because the cancer cells that proliferate after cancer occurrence have various genetic properties, these cancer cells develop slowly while cross talking with other cancer cells 
and surrounding cells. Cancer cells call on stromal cells in their neighborhoods, which are related with blood vessels and bloodvessel formation, to make their development advantageous and to facilitate metastasis, as well [11]. Thus, the stroma around cancer cells does not simply support those cancer cells but also actively assists cancer-cell growth, differentiation, and movement. SPARC interacts with extracellular matrix components such as collagens, laminin, fibronectin, and vitronectin and becomes involved in their metastasis [12, 13]. SPARC, as a material expressed in cancer cells and in the tissue cells around the cancer cells, is known to be related to the occurrence of various cancers. However, the research on SPARC expression in cases of colorectal cancer is very rare, though this cancer is on the increase in Korea. Therefore, this research used the immunohistochemical method to investigate the tissue microarray (TMA) SPARC expression for 332 colorectal cancer cases and determined if the result could be used as a prognosis prediction factor.

\section{METHODS}

\section{Materials}

We collected the tissues of 332 patients who underwent surgery for a colorectal carcinoma at Soonchunhyang University Cheonan Hospital over the decade from January 2000 to December 2010 and for whom paraffin blocks had been well preserved. We investigated gender, age, and radiologic inspection record on the basis of personal medical records, reviewed the tissue slides again for histological diagnoses, and determined the tumor $\mathrm{pT}$ and $\mathrm{pN}$ staging on the basis of the American Joint Committee on Cancer tumor staging policy.

\section{Methods}

For the fabrication of the TMAs, we reviewed the patients' tissue slides, collected two 2-mm cores from each paraffin block, and embedded them in a recipient block to plant 30 tissues per block. We then cut the TMA block into 4 - to 5 - $\mu$ m-thick pieces, stripped them of paraffin by using xylene 3 times for 5 minutes each time, and then treated them in ethanol of $100 \%, 95 \%, 90 \%, 70 \%, 50 \%$ and in distilled water for 3 minutes each until they became hydrous. For antigen retrieval, we put them in a $0.01-\mathrm{M}$ sodium-citrate buffer ( $\mathrm{pH} 6.0$ ), heated them in a microwave oven for $15 \mathrm{~min}$ utes, and cooled them for 30 minutes at room temperature. Then, we cleaned the tissue with phosphate-buffered saline (PBS, pH 7.4) solution, treated it with $0.3 \%$ hydrogen peroxide in methanol for 15 minutes at room temperature to suppress intrinsic peroxidase activity in the tissue, and finally cleaned it with distilled water. We washed the section with PBS, reacted it with goat sera for 30 minutes to block unspecific antigens, and removed the remaining solution. The primary antibody, SPARC mouse monoclonal antibody (Proteintech Group Inc., Chicago, IL, USA), was diluted to 1:100 and finally reacted at room temperature for 2 hours. Then, we flushed the section with PBS 3 times for 5 minutes each time and reacted it with primary antibody enhancer (Biovision, Milpitas, CA, USA, USA) at room temperature for 30 minutes. Thereafter, we repeated the flushing with PBS 3 times for 5 minutes each time, reacted it with polymer (Biovision) at room temperature for 30 minutes, and repeated the flushing with PBS 3 times for 5 minutes each time.

We used diaminobenzidine for color formation, used Mayer's hematoxylin for light contrast staining, used graded ethanol for dehydration, used xylene for transparency, used balsam for sealing, and used an optical microscope for observation. For immunohistochemical staining, we graded the cytoplasmic stain intensity as 0 to 3 ( 0 , unstained; 1 , mild staining; 2 , moderate staining; and 3 , severe staining) and classified the range as 1,2 , and $3(1$, less than $10 \%$; 2 , from $10 \%$ to $50 \%$; and 3 , from $50 \%$ to $100 \%$ ). If the sum of the intensity and the range exceeded 4 , it was read as positive, and we conducted a statistical processing. We applied the same rule to the stromal cell; i.e., we graded the stain intensity as 0 to 3 ( 0 , unstained; 1 , mild staining; 2 , moderate staining; and 3 , severe staining) and classified the range as 1,2 , and 3 (1, less than $10 \%$; 2 from $10 \%$ to $50 \%$; and 3 , from $50 \%$ to $100 \%$ ). If the sum of the intensity and the range exceeded 4 , it was read as positive, and we conducted a statistical processing.

\section{Statistical analysis of data}

We conducted the statistical validation in three groups: In the first group, SPARC was expressed in tumors, in the second, SPARC was expressed both in the tumor cell and the stroma, and in the third, SPARC was expressed in the stroma. For the interrelation between SPARC expression and the clinical and pathological variables, we used the $\chi^{2}$ test and the Student $t$ test. For the variation in survival according to SPARC expression, we used Kaplan-Meier survival curves, and for the influence of the clinical and pathological variables on the survival, we used the Cox proportional hazards model. For all statistical analyses, we used the SPSS ver. 14.0 (SPSS Inc., Chicago, IL, USA), and for all validations, we used the 2 -sided test, with $\mathrm{P}<0.05$ being considered as statistically meaningful.

\section{RESULTS}

The SPARC expression rates at the tumor, the stroma, and the tumor and stroma were 28\% (93/332), 46\% (154/332), and 22\% (73/ 332) respectively. No SPARC expression was observed in normal cells or their surrounding stromae. The SPARC expression in the stroma was much stronger than that in the tumor cells. The endothelial cells of the blood vessels and stromal fibroblasts around the tumor expressed SPARC strongly. The SPARC expressed as a glandular secreting-type expression, being conglomerated in the glandular lumen (Fig. 1).

According to the Cox proportional hazard regression analysis, the mortality risk was increased 2.10 times (95\% confidence interval, 1.05 to $4.19 ; \mathrm{P}=0.036$ ) when the tumor expressed SPARC. 
Volume 29, Number 3, 2013

Ann Coloproctol 2013;29(3):93-99 Coloproctology
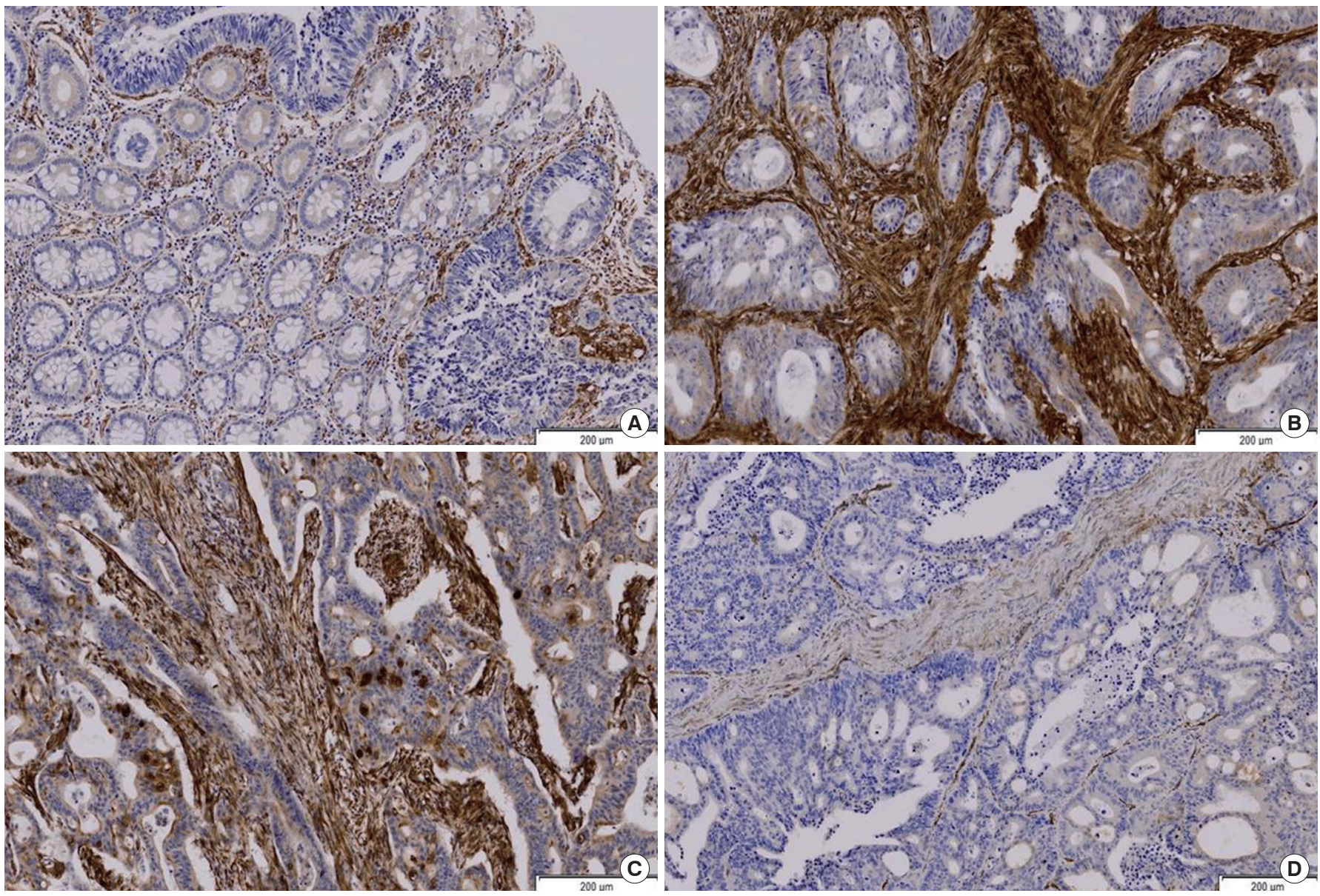

Fig. 1. (A) Secreted protein acidic and rich in cysteine (SPARC) is expressed mainly in the peritumor stroma (SPARC IHC, peroxidase, DAB). Mild expressions of SPARC in the tumor cells are noted while normal cells do not express SPARC. (B) SPARC is expressed strongly in the peritumor stroma. The tumor cells show moderate SPARC expression (SPARC IHC, peroxidase, DAB). (C) SPARC is expressed strongly in the peritumor stroma (SPARC IHC, peroxidase, DAB). The tumor cell expresses SPARC as a secretory component in the lumen. (D) SPARC is expressed neither in the stroma nor in the tumor (SPARC IHC, peroxidase, DAB).

Table 1. Cox regression analysis of the colorectal cancer with SPARC expression in tumor and clinicopathologic factors

\begin{tabular}{lcc}
\hline Clinicopathologic factor & Hazard ratio $(95 \% \mathrm{Cl})$ & P-value \\
\hline Age & $1.01(0.98-1.03)$ & 0.709 \\
Sex $(0=$ male, $1=$ female) & $0.71(0.36-1.40)$ & 0.326 \\
pT stage $(0=$ below $2,1=$ over 3$)$ & $2.67(0.32-22.62)$ & 0.368 \\
pN stage $(0=0,1=1,2)$ & $1.82(0.92-3.59)$ & 0.083 \\
Vascular invasion & $0.72(0.23-2.30)$ & 0.584 \\
Lymphatic invasion & $3.11(1.19-8.11)$ & 0.020 \\
SPARC expression in tumor $(0=-, 1=+)$ & $2.10(1.05-4.19)$ & 0.036 \\
\hline
\end{tabular}

SPARC, secreted protein acidic and rich in cysteine; $\mathrm{Cl}$, confidence interval.

In addition, in cases of lymphatic invasion, the mortality risk was increased by 3.11 times (95\% confidence interval, 1.19 to 8.11 ) in comparison with cases without lymphatic invasion, indicating that
Table 2. Cox regression analysis of the colorectal cancer with SPARC expression in stroma with clinicopathologic factors

\begin{tabular}{lcc}
\hline Clinicopathologic factor & Hazard ratio $(95 \% \mathrm{Cl})$ & P-value \\
\hline Age & $1.00(0.97-1.02)$ & 0.800 \\
Sex $(0=$ male, $1=$ female) & $0.61(0.31-1.22)$ & 0.165 \\
pT stage $(0=$ below $2,1=$ over 3$)$ & $3.28(0.37-28.77)$ & 0.283 \\
pN stage $(0=0,1=1,2)$ & $2.23(1.11-4.48)$ & 0.024 \\
Vascular invasion & $0.76(0.25-2.37)$ & 0.637 \\
Lymphatic invasion & $2.65(0.96-7.28)$ & 0.056 \\
SPARC expression (in stroma) $(0=-, 1=+)$ & $3.27(1.49-7.17)$ & 0.003 \\
\hline
\end{tabular}

SPARC, secreted protein acidic and rich in cysteine; $\mathrm{Cl}$, confidence interval.

the mortality risk was raised significantly $(\mathrm{P}=0.020)$ (Table 1$)$. According to the regression analysis, the mortality risk when the stromal cell expressed SPARC was significantly higher than the 
mortality risk when it did not by 3.27 times ( $95 \%$ confidence interval, 1.49 to $7.17 ; \mathrm{P}=0.003$ ) (Table 2). Also, in cases with lymphatic metastasis, the mortality risk increased by 2.23 times ( $95 \%$ confidence interval, 1.11 to 4.46 ), indicating a significantly increased mortality risk $(\mathrm{P}=0.024)$ (Table 2$)$.

According to the Cox proportional hazard regression analysis in

Table 3. Cox regression analysis of the colorectal cancer with SPARC expression both in tumor and in stroma and clinicopathologic factors

\begin{tabular}{lcc}
\hline Clinicopathologic factor & Hazard ratio $(95 \% \mathrm{Cl})$ & P-value \\
\hline Age & $1.00(0.98-1.03)$ & 0.755 \\
Sex $(0=$ male, $1=$ female) & $0.35(0.31-1.38)$ & 0.304 \\
pT stage $(0=$ below $2,1=$ over 3$)$ & $2.94(0.34-25.13)$ & 0.324 \\
pN stage $(0=0,1=1,2)$ & $1.90(0.97-3.73)$ & 0.062 \\
Vascular invasion & $0.77(0.23-2.56)$ & 0.667 \\
Lymphatic invasion & $2.79(1.04-7.48)$ & 0.042 \\
SPARC expression in tumor and stroma & $2.12(1.04-4.29)$ & 0.038 \\
\hline
\end{tabular}

SPARC, secreted protein acidic and rich in cysteine; $\mathrm{Cl}$, confidence interval.
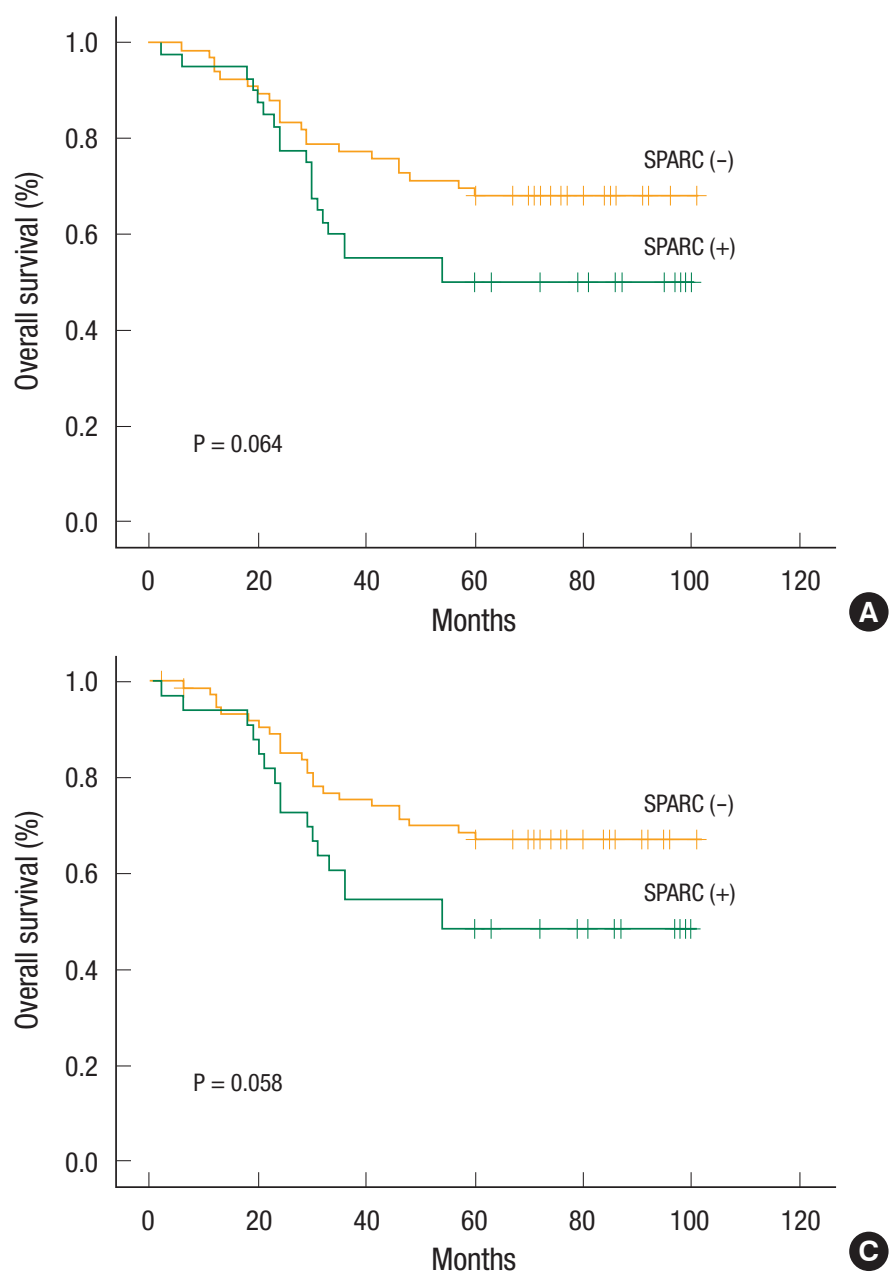

which we restricted the effects of age, gender, $\mathrm{pT}$ stage, $\mathrm{pN}$ stage, vascular invasion, lymphatic invasion, and differentiation, the mortality risk when SPARC was expressed both at tumors and stromal cells was significantly higher than the mortality risk when it was not by 2.12 times ( $95 \%$ confidence interval, 1.04 to 4.29$)(\mathrm{P}=$ 0.038 ). In addition, in cases with lymphatic invasion, the mortality risk was increased by 2.79 times ( $95 \%$ confidence interval, 1.01 to 7.48) in comparison with the cases without lymphatic invasion, indicating that the mortality risk was significantly increased $(\mathrm{P}=$ 0.042) (Table 3).

When we investigated the relation between SPARC expression and cumulative survival rate on the basis of the log-rank test result, we found no statistically significant difference in the cumulative survival rates whether SPARC was expressed in the tumor or not $(\mathrm{P}=0.064)$ (Fig. 2A). This phenomenon was also applicable to cases in which SPARC was expressed in both the tumors and the stromal cells simultaneously $(\mathrm{P}=0.058)$ (Fig. 2B). However, a statistically significant difference in cumulative survivals was found when SPARC was expressed in stromal cells $(\mathrm{P}=0.016)$ (Fig. 2C).

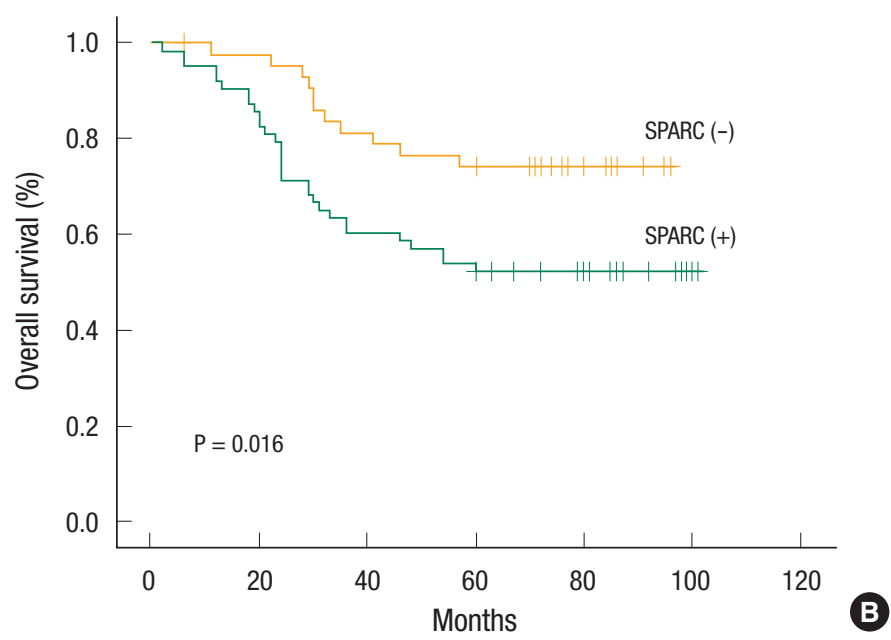

Fig. 2. (A) Survival curves from the time of diagnosis for colorectal cancer patients according to secreted protein acidic and rich in cysteine (SPARC) expression in the tumor ( $\mathrm{P}=0.064$, log-rank test). (B) Survival curves from the time of diagnosis for colorectal cancer patients according to SPARC expression in the stroma $(\mathrm{P}=0.016$, logrank test). (C) Survival curves from the time of diagnosis for colorectal cancer patients according to SPARC expression in both the tumor and the stroma $(\mathrm{P}=0.058$, log-rank test $)$. 


\section{DISCUSSION}

SPARC is a matricellular protein that plays an important role in the interaction between cells and substrates. This protein is a bonespecific phosphoprotein bonded to a collagen fibril and hydroxyapatite, and it was discovered by Termine et al. [14] in 1981. Thereafter, the same protein, which is secreted from blood-vessel stromal cells, was known as albumin-binding glycoprotein [15]. Until now, 6 kinds of proteins similar to SPARC have been identified, including testican-1, -2, -3, SPARC-like 1 (or hevin, Mast9), and SPARC-related modular calcium binding-1, -2 . The size of SPARC is $32 \mathrm{kDa}$, and although it consists of 303 amino acids, it is finally composed of 286 amino acids because the first 17 amino acids are missing. Because it is known to be expressed in bones and in bloodvessel stromal cells, it is expressed in a mass in notochord, somite, and gemmule bone during generation and is protein structures are maintained during evolution. Thus, it is known to play an important role in the physiological mechanism to maintain life phenomena $[16,17]$. Through genetic expression profiling of various malignant tumors, SPARC is known to be associated not only with malignant tumor development but also with poor prognosis. In particular, the melanoma expressing SPARC is related to an epithelial mesenchymal transition (EMT) to weaken the bond between the melanoma cell and the other cells due to the reduction of Ecadherin, thus reducing conjugation and converting the melanoma cell into a phenotype with a mesenchymal fibroblast aspect. Therefore, these cells are known to intensify cell invasion $[18,19]$.

Research on SPARC expression in cases of colorectal cancer in Korea was conducted for the first time by Yang et al. [10] in 2007. According to that research, the SPARC promoter methylation reduced the expression in most colorectal cancer cells, and its expression was useful for obtaining a prognosis. They also reported that SPARC was even expressed in normal colon tissue. However, according to that research, SPARC protein expression was found in the colorectal cancer cell lines HCT116, LoVo, SW480, HT29, and Colo205, but not in the Caco-2 cell line and not in normal cell lines like CCD-841-CoTr. Besides, SPARC expression was not found in most normal colorectal mucosal cells. It may be assumed that such a difference might have been caused by the different antibodies used.

The expression of SPARC in tumor tissues appeared weaker than that in the stromal cells surrounding the tumors. The stromal cell around the tumor is known to have interrelation with epithelial cells to help tumor generation. SPARC is strongly expressed not only in tumor cells but also in the surrounding stromal cells, in which case the prognosis is known to be poor because tumor infiltration and metastasis are promoted [20,21]. According to that research, SPARC was more strongly expressed in the stromal cells around the tumor cells but not in the stromal cells around normal mucous epithelia. In addition, it was expressed mildly in most tumor cells and strongly in the stromal cells around the tumor tissue, so it has not been proven the tumor cell provokes SPARC expres- sion in the surrounding stromal cells or that the surrounding stromal cells express SPARC to help the tumor cells to have invasive potentiality. Therefore, we need further research on this issue.

The EMT is an essential process in the formation of intermediate germ layer tissues like muscles and frames, in which at the time of normal gemmule growth, the epithelial cell loses its polarity, but has the epithelial-mesenchymal phenotype, causing the epithelial cell to transition to an epithelial-mesenchymal cell. This phenomenon can be easily observed in the process in which malignant cells are converted into a phenotype by infiltration into surrounding tissues and metastasis [22]. During tumor EMT, some changes, including reductions of inter-cell conjugation materials (E-N cadherin switching) and of epithelial cell indicators (cytokeratins), increases of the mesenchymal indicator vimentin, fibroblast-shape movability, and invasion-phenotype acquisition, occur. What is interesting is that the melanoma cell that expresses SPARC induces the EMT-related transcription factor snail expression [23]. In addition, deterioration of SPARC expression reduces $\mathrm{N}$-cadherin bonding with the cell membrane and water-soluble N-cadherin, as well [18]. In short, SPARC converts E-cadherin into Ncadherin to weaken cellular coupling and promotes malignant tumor infiltration and metastasis. On the other hand, when SPARC is reduced, $\mathrm{N}$-cadherin is converted into E-cadherin, intensifying the cellular coupling. Such a function of SPARC is quite obvious when we look into melanoma cells. In other malignant tumors except melanomas, the SPARC expression is related to tumor development. In metaplastic breast cancer, among breast cancers, whose development is very fast, EMT-related protein expression, including SPARC expression, is increased [2].

Under this research, as SPARC was expressed strongly not only in the tumor cell but also in its surrounding stromal cell, for statistical validation, we evaluated the clinicopathologic factors and placed the expressions in three groups: expressions in the tumor, in the stromal cells, and in both the tumor and the stromal cell simultaneously. This is the first study of the interrelation of the clinicopathologic factors with colorectal cancer by classifying SPARC expression into three groups: expression in the tumor, in the stromal cell, and in both the tumor and the stromal cell simultaneously. However, nothing was noteworthy, except the sexual difference, regarding the interrelation between the clinicopathologic factors and SPARC expression when SPARC expression was observed in the tumor cells. However, regarding the relation with survival on the basis of the Cox regression analysis, SPARC expression appeared to have a relation to survival; in particular, lymphatic invasion was revealed to affect survival.

Regarding SPARC expression in the tumor, E-cadherin reduction in melanoma cells is assumed to intensify invasion as well as lymphatic invasion. However, Yang et al's research result [10] that the tumor SPARC expression reduced cancer metastasis and prolonged the survival period contradicts this research result. Some reports said the SPARC expression was effective for cancer occurrence suppression while others said it contributed not only to can- 
cer occurrence but also to promotion of cancer development at the same time, so further research should be conducted on this subject. In case when SPARC is expressed in stromal cells, SPARC expression in the stromal cell, rather than SPARC expression in tumor cells, is assumed to be related to tumor-cell infiltration. When we consider that SPARC is an EMT-related material, SPARC expression in tumor stromal cells indicates that it is closely related to tumor cell invasion. According to the Cox regression analysis, SPARC expression in stromal cells is related to increased mortality risk $(\mathrm{P}=0.003)$, and the factor affecting survival was $\mathrm{pN}(\mathrm{P}=$ 0.024). Because SPARC tumor expression in stromal cells promotes cancer metastasis, it is assumed to be related to $\mathrm{pN}$. When SPARC expression appeared in both tumor cells and stromal cells simultaneously, SPARC expression was found to intensify cancer cell invasion. According to the Cox regression analysis, SPARC expression at tumors and stromal cells was related to increased mortality rate $(\mathrm{P}=0.038)$. Lymphatic invasion $(\mathrm{P}=0.042)$ also appeared to be related to it, and SPARC expression was closely related to the EMT, causing frequent lymphatic invasion. According to this research on the basis of SPARC expression, colorectal-carcinoma SPARC expression affected the survival rate, whether is the expression was in a tumor cell or in a stromal cell.

The recent tumor research is focused on tumor cell infiltration and metastasis caused by interactions with stromal cells in the neighborhood of tumor cells. Malignant tumors with fast development are related to SPARC expression, so some trials are being conducted to determine treatment by using SPARC expression. Tumor cell growth has been reported to be fundamentally blocked when melanoma SPARC expression at the time of melanoma injection into nude mice was appressed [24]. In another report, the growth of tumor cells injected in nude mice was suppressed when SPARC expression was decreased by applying adenovirus and nonviral plasmid vector to tumor cells $[18,25]$. In addition, tumor cell movement and infiltration were suppressed by applying antisense RNA in cases of breast cancer to suppress SPARC expression [26]. As shown above, much research is being conducted to study the cancer treatment method by using cancer progress suppression and setting SPARC expression to the treatment target. As malignant tumor SPARC expression is emerging as the target for early detection, prognosis prediction, and treatment, relevant research should follow.

In conclusion, tumor or stromal cell SPARC expression in patients with a colorectal carcinoma was closely related with colorectal cancer prognosis. Therefore, SPARC expression in a colorectal carcinoma is expected to be useful as a prognostic factor.

\section{CONFLICT OF INTEREST}

No potential conflict of interest relevant to this article was reported.

\section{REFERENCES}

1. Bornstein P, Sage EH. Matricellular proteins: extracellular modulators of cell function. Curr Opin Cell Biol 2002;14:608-16.

2. Lien HC, Hsiao YH, Lin YS, Yao YT, Juan HF, Kuo WH, et al. Molecular signatures of metaplastic carcinoma of the breast by large-scale transcriptional profiling: identification of genes potentially related to epithelial-mesenchymal transition. Oncogene 2007;26:7859-71.

3. Jones C, Mackay A, Grigoriadis A, Cossu A, Reis-Filho JS, Fulford L, et al. Expression profiling of purified normal human luminal and myoepithelial breast cells: identification of novel prognostic markers for breast cancer. Cancer Res 2004;64:3037-45.

4. Ledda F, Bravo AI, Adris S, Bover L, Mordoh J, Podhajcer OL. The expression of the secreted protein acidic and rich in cysteine (SPARC) is associated with the neoplastic progression of human melanoma. J Invest Dermatol 1997;108:210-4.

5. Rempel SA, Golembieski WA, Ge S, Lemke N, Elisevich K, Mikkelsen T, et al. SPARC: a signal of astrocytic neoplastic transformation and reactive response in human primary and xenograft gliomas. J Neuropathol Exp Neurol 1998;57:1112-21.

6. Yiu GK, Chan WY, Ng SW, Chan PS, Cheung KK, Berkowitz RS, et al. SPARC (secreted protein acidic and rich in cysteine) induces apoptosis in ovarian cancer cells. Am J Pathol 2001;159:609-22.

7. Puolakkainen PA, Brekken RA, Muneer S, Sage EH. Enhanced growth of pancreatic tumors in SPARC-null mice is associated with decreased deposition of extracellular matrix and reduced tumor cell apoptosis. Mol Cancer Res 2004;2:215-24.

8. DiMartino JF, Lacayo NJ, Varadi M, Li L, Saraiya C, Ravindranath Y, et al. Low or absent SPARC expression in acute myeloid leukemia with MLL rearrangements is associated with sensitivity to growth inhibition by exogenous SPARC protein. Leukemia 2006; 20:426-32.

9. Cheetham S, Tang MJ, Mesak F, Kennecke H, Owen D, Tai IT. SPARC promoter hypermethylation in colorectal cancers can be reversed by 5-Aza-2'deoxycytidine to increase SPARC expression and improve therapy response. Br J Cancer 2008;98:1810-9.

10. Yang E, Kang HJ, Koh KH, Rhee H, Kim NK, Kim H. Frequent inactivation of SPARC by promoter hypermethylation in colon cancers. Int J Cancer 2007;121:567-75.

11. Liotta LA, Kohn EC. The microenvironment of the tumour-host interface. Nature 2001;411:375-9.

12. Rosenblatt S, Bassuk JA, Alpers CE, Sage EH, Timpl R, Preissner KT. Differential modulation of cell adhesion by interaction between adhesive and counter-adhesive proteins: characterization of the binding of vitronectin to osteonectin (BM40, SPARC). Biochem J 1997;324(Pt 1):311-9.

13. Xie RL, Long GL. Role of N-linked glycosylation in human osteonectin. Effect of carbohydrate removal by N-glycanase and sitedirected mutagenesis on structure and binding of type $\mathrm{V}$ collagen. J Biol Chem 1995;270:23212-7.

14. Termine JD, Kleinman HK, Whitson SW, Conn KM, McGarvey 
ML, Martin GR. Osteonectin, a bone-specific protein linking mineral to collagen. Cell 1981;26(1 Pt 1):99-105.

15. Sage H, Johnson C, Bornstein P. Characterization of a novel serum albumin-binding glycoprotein secreted by endothelial cells in culture. J Biol Chem 1984;259:3993-4007.

16. Holland PW, Harper SJ, McVey JH, Hogan BL. In vivo expression of mRNA for the Ca++-binding protein SPARC (osteonectin) revealed by in situ hybridization. J Cell Biol 1987;105:473-82.

17. Mason IJ, Murphy D, Munke M, Francke U, Elliott RW, Hogan BL. Developmental and transformation-sensitive expression of the Sparc gene on mouse chromosome 11. EMBO J 1986;5:1831-7.

18. Sosa MS, Girotti MR, Salvatierra E, Prada F, de Olmo JA, Gallango SJ, et al. Proteomic analysis identified N-cadherin, clusterin, and HSP27 as mediators of SPARC (secreted protein, acidic and rich in cysteines) activity in melanoma cells. Proteomics 2007;7: 4123-34.

19. Smit DJ, Gardiner BB, Sturm RA. Osteonectin downregulates Ecadherin, induces osteopontin and focal adhesion kinase activity stimulating an invasive melanoma phenotype. Int J Cancer 2007; 121:2653-60.

20. Infante JR, Matsubayashi H, Sato N, Tonascia J, Klein AP, Riall TA, et al. Peritumoral fibroblast SPARC expression and patient outcome with resectable pancreatic adenocarcinoma. J Clin Oncol 2007;25:319-25.
21. Koukourakis MI, Giatromanolaki A, Brekken RA, Sivridis E, Gatter KC, Harris AL, et al. Enhanced expression of SPARC/osteonectin in the tumor-associated stroma of non-small cell lung cancer is correlated with markers of hypoxia/acidity and with poor prognosis of patients. Cancer Res 2003;63:5376-80.

22. Thiery JP. Epithelial-mesenchymal transitions in tumour progression. Nat Rev Cancer 2002;2:442-54.

23. Robert G, Gaggioli C, Bailet O, Chavey C, Abbe P, Aberdam E, et al. SPARC represses E-cadherin and induces mesenchymal transition during melanoma development. Cancer Res 2006;66:7516-23.

24. Ledda MF, Adris S, Bravo AI, Kairiyama C, Bover L, Chernajovsky Y, et al. Suppression of SPARC expression by antisense RNA abrogates the tumorigenicity of human melanoma cells. Nat Med 1997;3:171-6.

25. Alvarez MJ, Prada F, Salvatierra E, Bravo AI, Lutzky VP, Carbone $\mathrm{C}$, et al. Secreted protein acidic and rich in cysteine produced by human melanoma cells modulates polymorphonuclear leukocyte recruitment and antitumor cytotoxic capacity. Cancer Res 2005; 65:5123-32.

26. Briggs J, Chamboredon S, Castellazzi M, Kerry JA, Bos TJ. Transcriptional upregulation of SPARC, in response to c-Jun overexpression, contributes to increased motility and invasion of MCF7 breast cancer cells. Oncogene 2002;21:7077-91. 